\title{
Fabrication of a Nanocomposite Based on Pectin/ Polyaniline/Graphene Oxide as Novel Electrically Conductive Biomaterial for Biosensing Detection of Hemoglobin
}

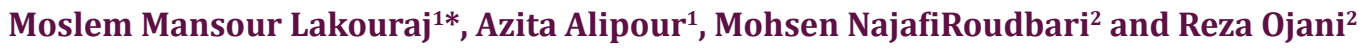 \\ ${ }^{1}$ Department of Organic Chemistry, Faculty of Chemistry, University of Mazandaran, Iran
}

${ }^{2}$ Department of Analytical Chemistry, Faculty of Chemistry, University of Mazandaran, Iran

*Corresponding author: Moslem Mansour Lakouraj, Polymer Laboratory, Department of Organic Chemistry, Faculty of Chemistry, University of Mazandaran, P.O. Box 47416, Babolsar, Iran

\begin{abstract}
A novel biomaterial based on hydrolyzed pectin(HP)/polyaniline(PA)/graphene oxide(GO) has been prepared via chemical polymerization method. The SEM analyses revealed a rod-like and laminated morphology for NH-mGO@HP and NH-mGO@HPPA nanocomposites. Higher thermal stability for NH-mGO@HP-PA was obtained than other samples. Biosensor was designed via electrodeposition of NH-mGO@HP-PA nanocomposite on carbon graphite electrode. Biosensor activity showed a considerable increase in oxidation current by the nanocomposite. The NH-mGO@HP-PA indicated higher electrical conductivity compared to pure PA.
\end{abstract}

Keywords: Polyaniline; Graphene oxide; Electrochemical properties; Hemoglobin biosensor

\section{Introduction}

Over the recent years, there have been many investigations on carbon nanomaterials namely graphene oxide due to their high surface area, electrical and electrochemical properties. GO is a single sheet of graphite oxide that because of a wide range of functional groups including oxygen on its surface capable to be modified chemically with other materials [1-2].GO is recognized as an actual nanocarbon due to its superior mechanical, structural and thermal properties relative to other conventional nanomaterials. It can be synthesized via Hummer method [1,3]. PA/GO nanocomposites with improved electrochemical performance as energy storage and supercapacitor were reported in some studies [4-6]. However,there are few studies on PA/GO nanocomposites as electrocatalyst in biosensors [7-8]. The poor electrical conductivity of GO caused by the functional groups creating high electron transfer resistant, like carboxyl, hydroxyl and epoxy groups in its surface limited its direct application in electrochemical applications. However, the incorporation of graphene oxide in a conducting polymer matrix results in good mechanical strength and electrochemical properties
[9]. PA is one of the conducting polymers which have attracted much attention in sensor areas as excellent organic conductor with superior electrical and electrochemical properties, good chemical and environmental stability and biocompatibility [10]. The combination of PA with other nanomaterials can provide its application as a desirable matrix for immobilization and detection of biomolecules via charge transfer reactions [11-14]. Pectin is a biocompatible high-molecular weight polysaccharide extracted from cell walls of plants that is composed of three structures of homogalacturonan, rhamnogalacturonan I and rhamnogalacturonan II. In spite of its unique properties such as good gelling properties, biocompatibility, non-toxicity and biodegradability, it has some inherent flaws that make it unfavorable for application in some specific fields. The chemical modification of pectin was considered to remove these drawbacks. Due to the existence of structural groups such as hydroxyl and carboxyl group on its surface along the backbone, a wide range of pectin derivatives can be prepared via chemical modification [15]. In addition, pectin due to possessing 
functional groups including oxygen can provide a suitable medium for electron transfer between electrode and electrolyte solution.

In this work we present a nanocomposite hydrogel of $\mathrm{NH}$ mGO@HP-PA for biosensing detection of hemoglobin. In this work pectin was used as hydrolyzed pectin (HP). The NH-mGO@ HP-PA nanocomposite was synthesized via synthesis of GO with hummer method, modification of GO via amine treatment with diethylenetriamine (NH-mGO), immobilization of HP to NH-mGO by diisocyanate as spacer or coupling agent (NH-mGO@HP) and finally surface polymerization of PA on NH-mGO@HP to give NHmGO@HP-PA. The synthesized samples were examined in terms of structural, morphological and thermal properties via FTIR, XRD, SEM and TGA analyses. The NH-mGO@HP-PA nanocomposite was evaluated as biosensor for electrochemical detection of hemoglobin. This method is a facile, efficient, and green route for development of a biomaterial based on GO and PA for biological sensors.

\section{Experimental}

\section{Materials}

Graphite powder, potassium permanganate, sodium nitrate and hydrogen peroxide were provided from commercial source (Merck, Schuchardt Germany). Aniline was obtained from Merck and doubly distilled and purified before use to eliminate any oxidation products. Other chemicals containing diethylenetriamine (DETA), $\mathrm{H}_{2} \mathrm{SO}_{4}$ (98\%), $\mathrm{HCl}(37 \%), \mathrm{NaOH}$, diisocyanate, acetone and dimethylsolfoxide (DMSO) were acquired from Merck (Schuchardt Germany). Except aniline, other materials were used as received without purification. Ammonium persulfate $\left(\left(\mathrm{NH}_{4}\right)_{2} \mathrm{~S}_{2} \mathrm{O}_{8}\right)$ (APS), hemoglobin $(\mathrm{Hb})$ and glutaraldehyde (GA) were purchased from Asia Pajohesh Company (ltd). Pepsin enzyme was provided from? Pectin was bought from Sigma-Aldrich.

\section{Characterizations}

The samples for X-ray diffraction (XRD) analysis were studied by a Philips PW1730 X-ray diffractometer (Netherlands) at a scan rate of $10^{\circ} \mathrm{C} / \mathrm{min}$. A FESEM TESCAN MIRA Пdevice (Czech) was utilized to perform scanning electron microscopy (SEM) analysis and to observe the surface morphology of the samples. Fourier transform infrared spectroscopy (FTIR) was used for structural characterization of the samples. The diffraction patterns were recorded using a BRUKER TENSOR27 FTIR spectrometer (Germany), in the range of $400-4000 \mathrm{~cm}^{-1}$ in form of $\mathrm{KBr}$ pellet. The thermal stability of the samples was attained by thermogravimetric analysis (TGA) with a TGA-DTA METTLER TGA/STTA851 (Switzerland) thermogravimetry under Ar atmosphere at a heating rate of $10^{\circ} \mathrm{C} / \mathrm{min}$ from 25 to $600^{\circ} \mathrm{C}$. The electrical conductivity was determined via a four-probe method with the sample prepared as pellet. The electrical conductivity is defined as the below equation:

$$
\sigma=\frac{\operatorname{In} 2}{\pi d} * \frac{I}{V}
$$

in which $\sigma$ is the conductivity $\left(\mathrm{S} \mathrm{cm}^{-1}\right)$, I is the applied current $(\mathrm{mA})$ through outer probes, $\mathrm{V}$ is the voltage drop $(\mathrm{mV})$ measured across the inner probes and $d$ is the thickness of sample $(0.1 \mathrm{~cm})$.

\section{Design of Biosensor and Electrochemical Measurement}

The biosensor was fabricated on a carbon graphite electrode (CGE) electrodeposited with NH-mGO@HP-PA nanocomposite and immobilized with pepsin enzyme. A CGE was prepared by graphite powder, paraffin oil, a capillary tube and a copper wire. $160 \mathrm{mg}$ of graphite powder was mixed with one drop of paraffin oil. This mixture was mixed in a borosilicate glass vial to form a homogeneous carbon graphite material. A copper wire was inserted into the capillary tube and carbon graphite mixture was manually packed into the capillary to give bare carbon graphite electrode. The obtained CGE was completely polished on a paper. The NHmGO@HP-PA nanocomposite was electrochemically deposited in an electrochemical cell on three electrodes containing $\mathrm{Ag} / \mathrm{AgCl}$ as the reference electrode, Pt as counter electrode and carbon graphite electrode as working electrode. After electrodeposition, the electrode was dried at room temperature to obtain a composite modified CGE (NH-mGO@HP-PA/CGE). Afterward, in order to the immobilization of enzyme and detection of $\mathrm{Hb}$, the composite modified CGE (NH-mGO@HP-PA/CGE) was dropped with an enzyme solution containing 4:1 of $\mathrm{Hb}$ to pepsin and a GA solution as binder with a ratio of 2:1 pepsin: GA. The electrochemical measurements were performed by a Biological SP150 electrochemical device in an acetate buffer solution containing $\left[\mathrm{Fe}(\mathrm{CN})_{6}\right]^{3-/ 4-}$ as electrolyte. The cyclic voltammetry was performed in the range of $500-700 \mathrm{mV}$ at different scan rates and electrodeposition was carried out for 30 $\min$ at $-600 \mathrm{mV}$.

\section{Synthetic Procedures}

Preparation of G0: GO was synthesized by a modified Hummer method according to previous report [1]. In a typical synthesis, a flask containing $3.0 \mathrm{~g}$ graphite $(3.0 \mathrm{~g}), 1.5 \mathrm{~g} \mathrm{NaNO}_{3}$ and $69 \mathrm{~mL} \mathrm{H}_{2} \mathrm{SO}_{4}$ was kept at the temperature below $5^{\circ} \mathrm{C}$. Subsequently $9.0 \mathrm{~g} \mathrm{KMnO}_{4}$ was added to the flask and the temperature was maintained below $20^{\circ} \mathrm{C}$. The reaction was continued for $7 \mathrm{~h}$ at $35^{\circ} \mathrm{C}$. After that, another amount of $9.0 \mathrm{~g} \mathrm{KMnO}_{4}$ was slowly added to the flask. The reaction system was reacted for $12 \mathrm{~h}$ at this temperature and cooled to room temperature then diluted by $400 \mathrm{~mL}$ of ice water and followed by the slow addition of $3 \mathrm{~mL}$ of $30 \%$ aq $\mathrm{H}_{2} \mathrm{O}_{2}$. The obtained dispersion was washed repeatedly with $\mathrm{H}_{2} \mathrm{O}$ and $\mathrm{HCl}$ to remove the remnant salt. Finally, the resulting precipitate was dried in an oven at $40^{\circ} \mathrm{C}$ for $24 \mathrm{~h}$.

Modification of GO: The amine modification of GO was proceeded via a moderate route by treatment with diethylenetriamine as amine modifying agent according to the prior research [1].A certain amount of GO and diethylenetriamine were loaded in $95 \%$ ethanol and sonicated for $40 \mathrm{~min}$. The reaction was performed for $24 \mathrm{~h}$ at room temperature. At last, the suspension was filtered, washed successively with ethanol, methanol and acetone then dried in an oven at $40^{\circ} \mathrm{C}$ overnight to give NH-mGO@HP.

Immobilization of HP on NH-mGO@HP: The modification of GO with HP was conducted by a diisocyanate as spacer agent. At first, pectin was hydrolyzed through alkaline method by a $\mathrm{NaOH}$ solution $(3 \mathrm{M})$ at $50^{\circ} \mathrm{C}$ for $72 \mathrm{~h}$ to get HP. The obtained solid product was 
collected with acetone and dried in an oven at $40^{\circ} \mathrm{C}$. To synthesize NH-mGO@HP, the synthesized HP (0.6g) was dispersed in $20 \mathrm{~mL}$ DMSO in a $100 \mathrm{~mL}$ round-bottom flask equipped with a magnetic stir bar under sonication for $30 \mathrm{~min}$, then diisocyanate $(1: 10)$ and $20 \% \mathrm{~W} / \mathrm{W}$ diethylenetriamine as catalyst were added into the above suspension and subsequently was reacted with a dispersion of $0.3 \mathrm{~g} \mathrm{NH}-\mathrm{mGO}$ in $20 \mathrm{~mL}$ DMSO under nitrogen atmosphere. The reaction was allowed to continue for $24 \mathrm{~h}$ at $70^{\circ} \mathrm{C}$ to twice as high efficiency. Finally, the obtained gelling product was poured into a petri dish and dried in an oven at $40^{\circ} \mathrm{C}$.

Preparation of NH-mGO@HP-PA nanocomposite: To synthesize this nanocomposite, a dispersion mixture of $\mathrm{NH}$ mGO@HP in distilled water was prepared via sonication then an aqueous solution of APS (molar ratio of 1:1) was introduced into the above dispersion. After $1 \mathrm{~h}$ stirring, the polymerization mixture was followed by the addition of an aniline solution with weight ratio of 1:2 (NH-mGO@HP: aniline) in $\mathrm{HCl}$ solution (1M). The polymerization was reacted for $24 \mathrm{~h}$ to be complete. The resultant crude product was filtered, washed with distilled water and acetone and dried in an oven at $40^{\circ} \mathrm{C}$ overnight. (Figure 1).

The synthetic path of the prepared samples is shown schematically in Figures2-4,(Schemes 1-3).

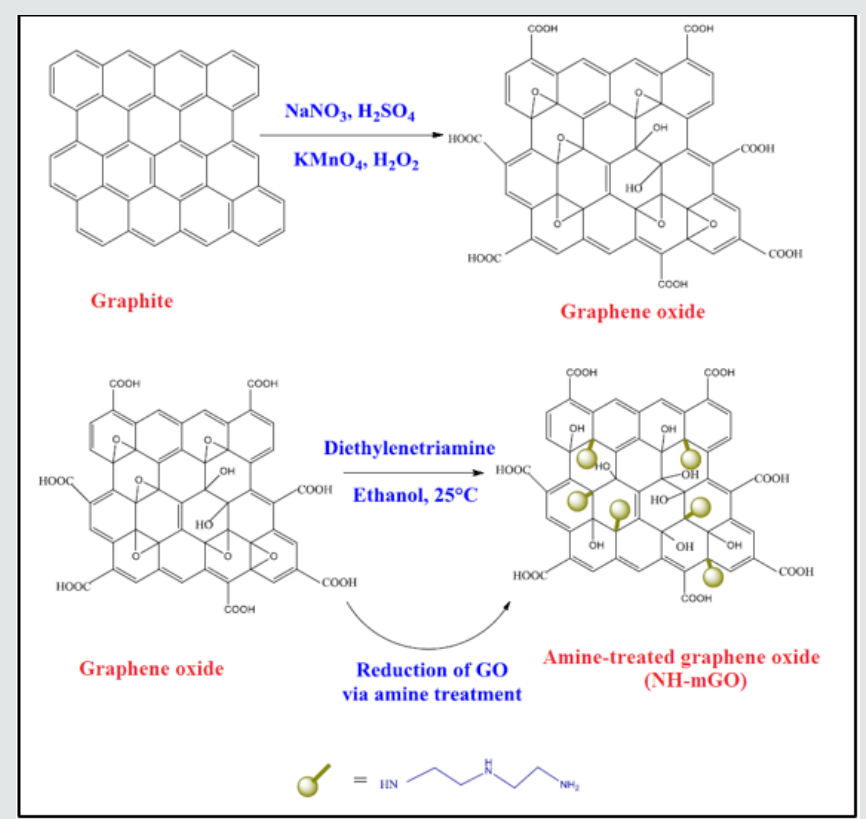

Scheme 1: The schematic illustration of the synthesis of GO and NH-mGO nanoparticles.

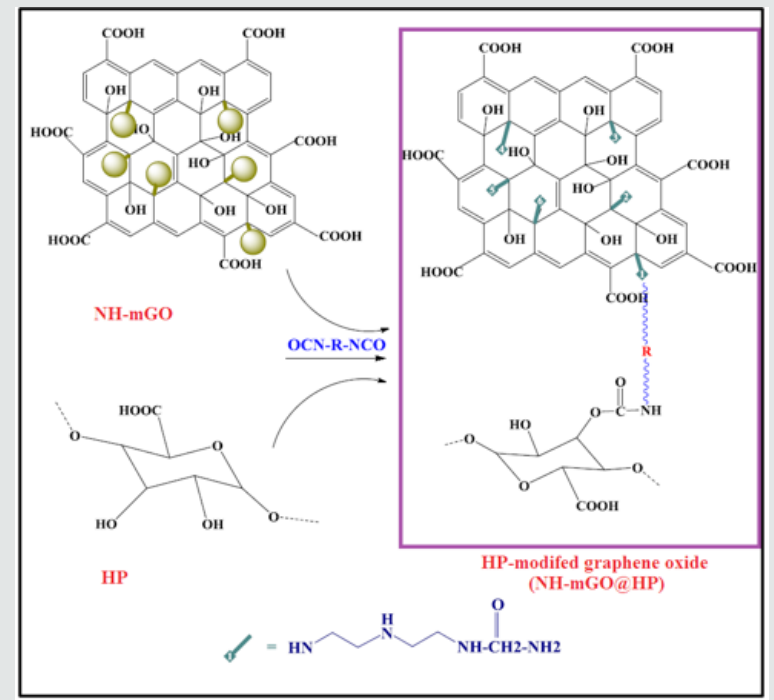

Scheme 2: The synthetic path of the NH-mGO@HP nanoparticle. 


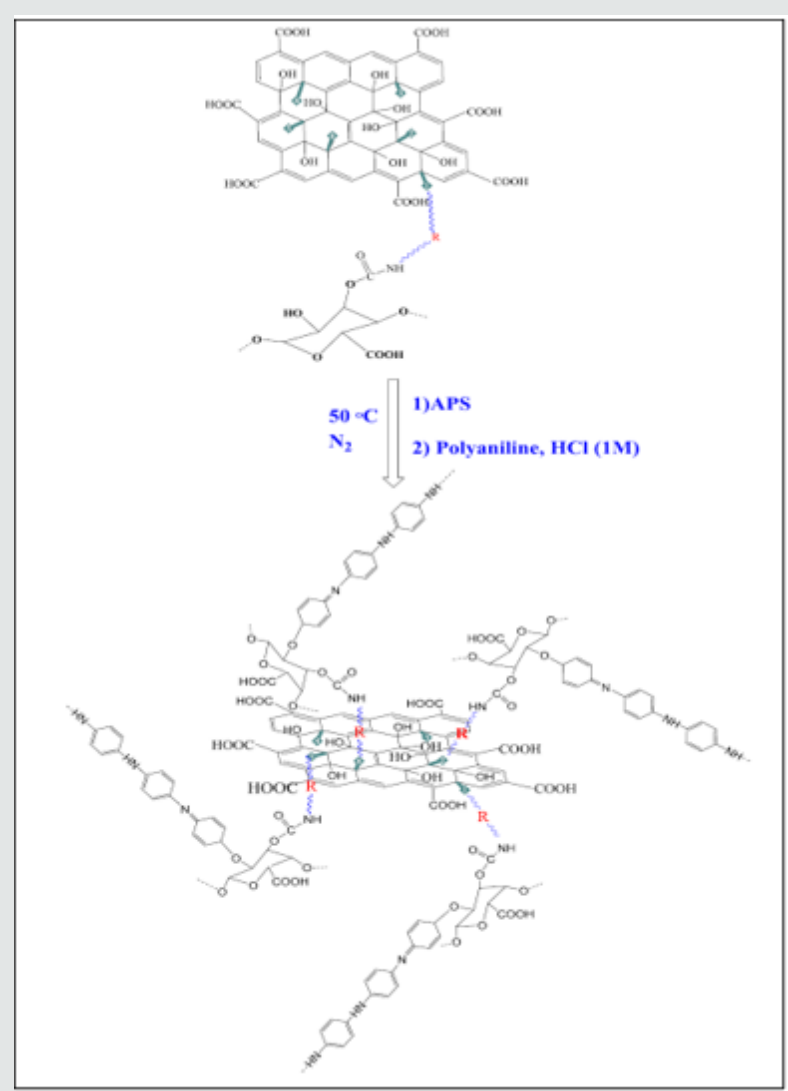

Scheme 3: The schematic representation of the synthetic path of the NH-mGO@HP-PA nanocomposite.

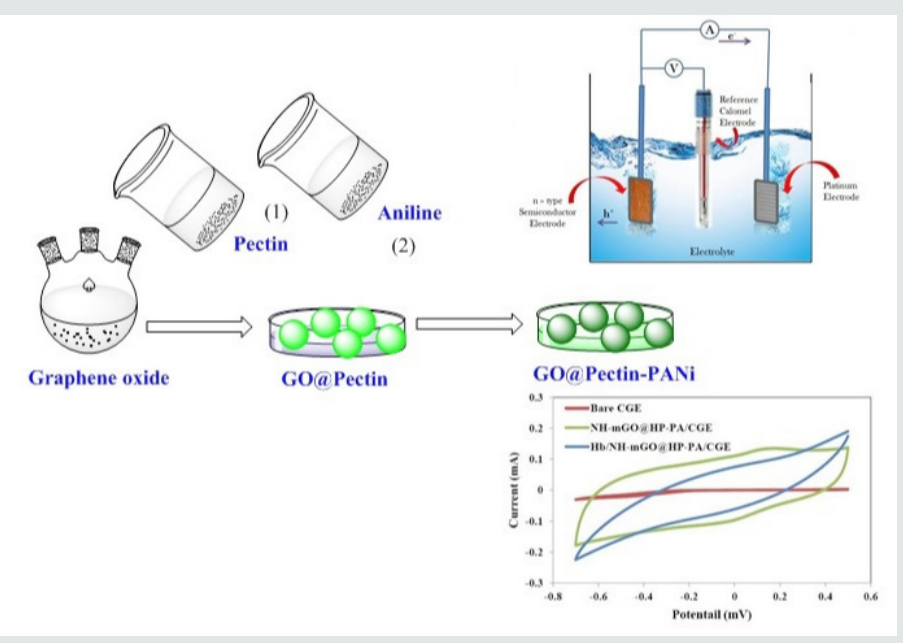

Figure 1: Graphical abstract.

\section{Result and Discussion}

\section{Structural Characterization}

The FT-IR spectra of the samples of GO, NH-mGO and NHmGO@HP are exhibited in (Figure2).As exhibited in (Figure2a), the IR spectrum of GO shows representative peaks at $\sim 1009-1168 \mathrm{~cm}$ 1, $\sim 1434,1571 \mathrm{~cm}^{-1}, \sim 1632,1712 \mathrm{~cm}^{-1}$ and $\sim 3437 \mathrm{~cm}^{-1}$ assigned to $\mathrm{C}-\mathrm{O}-\mathrm{C}$ stretching of epoxide rings, $\mathrm{C}=\mathrm{C}$ stretching of aromatic rings, $\mathrm{C}=\mathrm{O}$ stretching of carboxyl group, and $\mathrm{O}-\mathrm{H}$ stretching respectively
(1). The infrared absorption spectrum of NH-mGO (Figure2b) reveals new peaks at $\sim 2907-3251 \mathrm{~cm}^{-1}$ and also at $\sim 1123 \mathrm{~cm}^{-1}$ ascribed to $\mathrm{N}-\mathrm{H}$ stretching and $\mathrm{C}-\mathrm{N}$ stretching vibrations respectively that propose the GO treated with diethylenetriamine via ringopening reaction of epoxy groups. Furthermore the peaks related to $\mathrm{C}=\mathrm{O}, \mathrm{C}-\mathrm{O}-\mathrm{C}$ epoxy groups and $\mathrm{O}-\mathrm{H}$ stretching was decreased in the NH-mGO in comparison with GO that demonstrates the elimination of epoxy functional groups from the surface of GO in the NH-mGO. The modification of the NH-mGO with HP via the reaction 
with diisocyanate as a spacer agent can result in the creation of urethane and urea functional groups by means of edge carboxyl or surface hydroxyl groups of HP with amine groups of the NH-mGO respectively as can be seen in (Figure2c). The absorption changes happened upon the modification of GO with HP via diisocyanate treatment can be observed via the appearance of the bands at $\sim 1632 \mathrm{~cm}^{-1}$ and $\sim 1693 \mathrm{~cm}^{-1}$ which can be attributed to $\mathrm{C}=0$ stretching of urea functional groups and imine $\mathrm{NH}=\mathrm{C}$ stretching of urethane groups formed respectively. In addition, it is noteworthy that the absence of the peaks associated to the isocyanate groups (2275-2263 $\left.\mathrm{cm}^{-1}\right)$ suggesting the modification of GO and HP with diisocyanate. The main characteristic absorption peaks of PA segments in NH-mGO@HP-PA (Figure2d) were detected at $~ 1464$ $\mathrm{cm}^{-1}, \sim 1567 \mathrm{~cm}^{-1}, \sim 1288 \mathrm{~cm}^{-1}$ and $\sim 3430 \mathrm{~cm}^{-1}$ asigned to $\mathrm{C}=\mathrm{C}$ and $\mathrm{C}=\mathrm{N}$ stretching of benzenoid and quinoid structures, $\mathrm{C}-\mathrm{N}$ and $\mathrm{N}-\mathrm{H}$ stretching vibrations respectively.
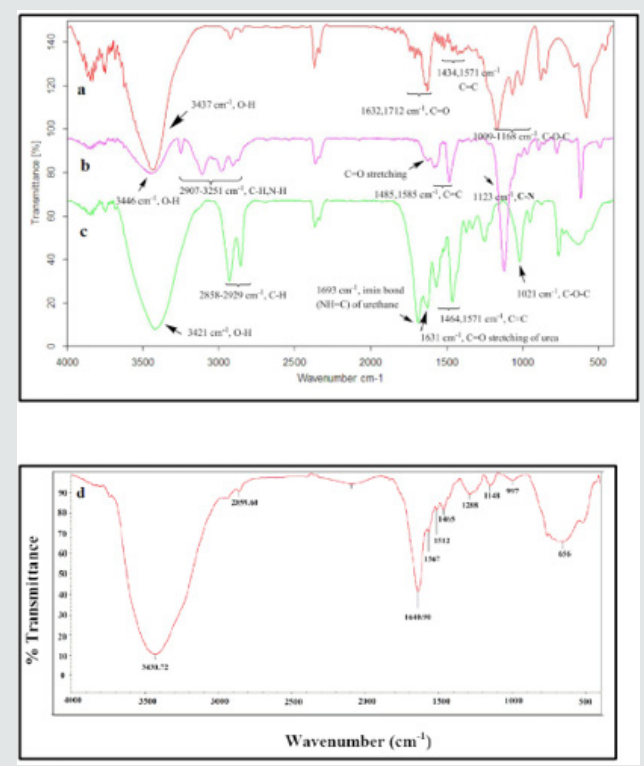

Figure 2: The FTIR spectra of a) GO, b) NH-Mgo, c) NH-mGO@HP and d) NH-mGO@HP-PA.

\section{Morphological Characterization}

The surface morphology of the samples was examined by the SEM analyses and the images are illustrated in (Figure3). The micrographs revealed a laminar-like morphology with stacked sheets for GO and nanorod-like morphology for NH-mGO@HP. This change in the morphology can corroborate the stabilization of the HP segments on the GO layers. For the NH-mGO@HP-PA nanocomposite, a typical globular-laminar morphology with embedded GO layers was observed in the SEM image. It can be realized from the micrographs that the coating of the PA on the surface of the NH-mGO@HP nanoparticles reduced the size of the nanoparticles from nanorod to globular shape.

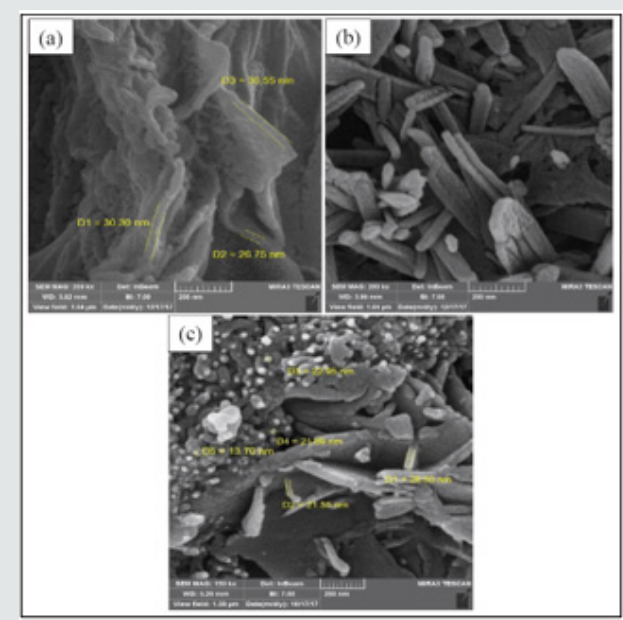

Figure 3: The SEM micrograph of the synthesized samples, a) GO, b) NH-mGO@HP and c) NH-mGO@HP-PA. 


\section{Crystalline Structure}

The crystalline structure of the samples was investigated via XRD experiments and the diffractograms are indicated in (Figure4). The XRD pattern of the synthesized GO shows a strong and sharp peak at $2 \theta=14^{\circ}$ corresponding to (001) reflection of G0 sheet (9) and peaks at $2 \theta=24^{\circ}$ and $42^{\circ}$ corresponding to (002) and (101) reflections respectively. For NH-mGO@ HP and NH-mGO@HPPA nanocomposites, the intensity of the diffraction peaks of GO at $2 \theta=14^{\circ}$ and $42^{\circ}$ was reduced. This reduction can be due to the exfoliation of the GO layers with the HP and PA segments. The peak at $2 \theta=23^{\circ}$ appeared with more intensity in these composites compared with GO which illustrates the linkage of HP and PA chains on the surface of GO. Therefore, regarding to the XRD patterns, the results demonstrated the well exfoliation of the GO layers, their desirable linkage to the polymeric composite matrix and a semicrystalline structure for the nanocomposite which may be slightly caused by the presence of the HP segments and also incorporation of GO.

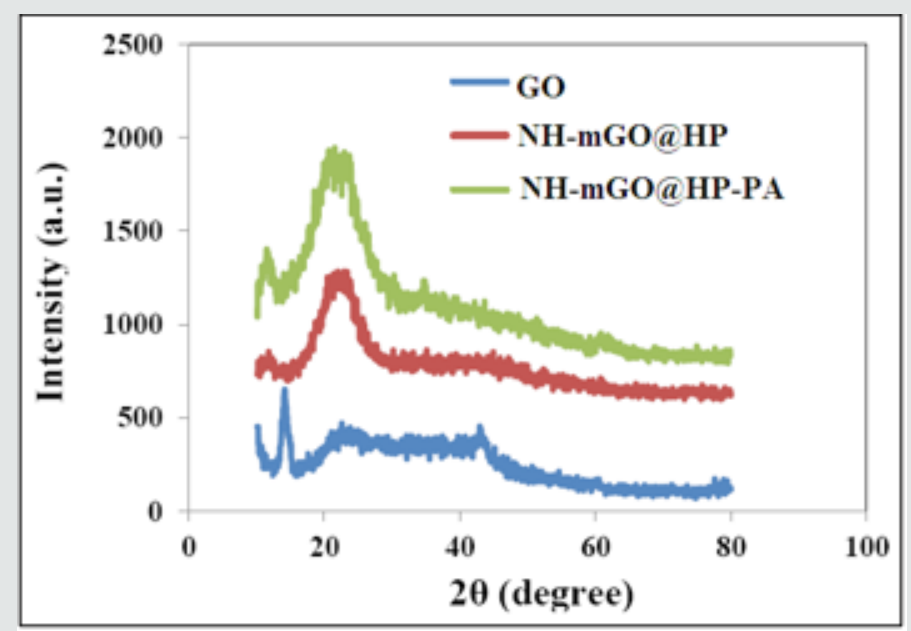

Figure 4: The XRD pattern of the prepared samples.

\section{Thermal Analysis}

The samples were examined in terms of thermal stability by TGA analysis and the results are shown as curve in (Figure5)and being summarized as comparative data in Table 1. The TGA curve of GO shows a weight loss at $73^{\circ} \mathrm{C}$ correspond to the removal of residual water. A weight loss occurred at $225^{\circ} \mathrm{C}$ attributed to the pyrolysis of the labile functional groups containing oxygen and as well the glycoside bonds in GO. The weight loss after $225^{\circ} \mathrm{C}$ is assigned to the skeletal degradation of the GO. For the sample of the NH-mGO@HP, the temperatures related to the removal of water, scission of the glycoside bonds and also degradation of composite backbone transferred to higher temperatures $\left(168^{\circ} \mathrm{C}, 339^{\circ} \mathrm{C}\right.$ and $449^{\circ} \mathrm{C}$ respectively) relative to GO. This transfer can be caused by the crosslinking connections existed between the HP segments and GO layers which restrict the free spaces available in the composite and also due to more functional groups containing oxygen that accordingly enhance such these thermal occurrences. The thermal degradation process of NH-mGO@HP-PA happened at higher temperature (at $472^{\circ} \mathrm{C}$ ) in comparison with the NH-mGO@HP.

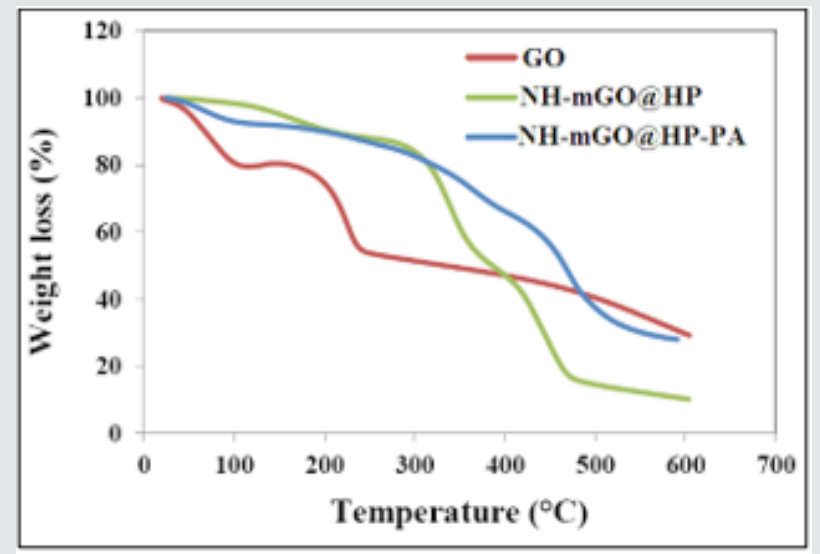

Figure 5: The TGA thermograms of the prepared samples. 
Table 1: The comparative TGA results of the samples.

\begin{tabular}{|c|c|c|}
\hline Sample & $\begin{array}{l}\text { Final Degradation } \\
\text { Temperature (Tf) }\end{array}$ & $\begin{array}{l}\text { Weight Retention (\%) } \\
\quad\left(\text { at } 600^{\circ} \mathrm{C}\right)\end{array}$ \\
\hline GO & 225 & 29.45 \\
\hline NH-mGO@HP & 449 & 10.2 \\
\hline NH-mGO@HP-PA & 472 & 28.04 \\
\hline
\end{tabular}

The scission of glycoside bonds was enhanced to $361^{\circ} \mathrm{C}$. Moreover, a weight loss was observed at $237^{\circ} \mathrm{C}$ assigned to the removal of dopant from the nanocomposite. These observations illustrate the influence of the PA chains in the heightening of the thermal stability. It is conceived from the results that with the stabilization of HP on GO in the NH-mGO@HP sample, the thermal stability increased because of the crosslinking linkages between the HP segments and GO layers and also exfoliation and dispersion of the GO layers in the nanocomposite matrix while the residual weight (\%) was reduced from $29.45 \%$ to $10.2 \%$. This reduction can be attributed to the presence of the glycosidic bonds of the HP molecules in the NH-mGO@HP that accelerates the decomposition of the HP segments and consequently the degradation of the composite. Subsequently the coating of the PA on the NH-mGO@ HP, raised the thermal stability and residual weight (\%) of the nanocomposite which is mainly due to the existence of a carbon net structure in the nanocomposite.

\section{Electrical Conductivity}

Table 2: The electrical conductivity results of the PA and $\mathrm{NH}-$ mGO@HP-PA.

\begin{tabular}{|c|c|}
\hline Sample & $\boldsymbol{\sigma}\left(\mathbf{S ~ c m}^{-}{ }^{\mathbf{}}\right)$ \\
\hline PA & 0.15 \\
\hline NH-mGO@HP-PA & 0.242 \\
\hline
\end{tabular}

The electrical conductivity was measured via four-probe method and results are presented in Table 2 . The results show that the presence of GO led to the increase of the conductivity.

\section{Solubility}

The sample was obtained as hydrogel due to high hydrophilicity of pectin. The solubility of the nanohydrogel is shown in (Figure6). As can be observed the biomaterial displayed high solubility in polar dimethylformamide solvent.

\section{Electrochemical Biosensing Measurement}

The electrochemical biosensing measurement was evaluated via cyclic voltammetry. The cyclic voltammetry responses of bare CGE, nanocomposite modified CGE (NH-mGO@HP-PA/CGE) and the NH-mGO@HP-PA/CGE after dropping of $\mathrm{Hb}$ was compared in (Figure7). As can be observed from voltammograms, the bare CGE didn't show any visible redox peaks while the NH-mGO@HP-PA/ CGE electrode indicated a considerable increase in redox peaks indicating the electrodeposition of NH-mGO@HP-PA nanocomposite on the surface of CGE. This considerable enhancement can be also attributed to the improved electron communication between electrode and electrolyte. After enzyme immobilization and dropping of $\mathrm{Hb}$ solution, a well-defined reversible redox peak with a reduction in the redox currents was observed due to the cover of pepsin enzyme to the PANi coating on the surface of electrode. This reduction is indicating the sensitivity of the $\mathrm{NH}$ mGO@HP-PA/CGE to the presence of biomolecule of Hb through the enzymatic detection. Indeed, this observation confirms the biosensing performance of the NH-mGO@HP-PA nanocomposite to detection of $\mathrm{Hb}$. In the cyclic voltammogram of NH-mGO@HP-PA/ CGE, the reduction and oxidation occurred at -0.1 and $0.1 \mathrm{~V}$, which are almost equal, and the redox curves are almost symmetric. The ratio of the redox currents (Ipa/Ipc) was about 1 proposing that the electrochemical reaction is reversible. The plausible mechanism of biosensor activity of the nanocomposite is shown in(Figure8).

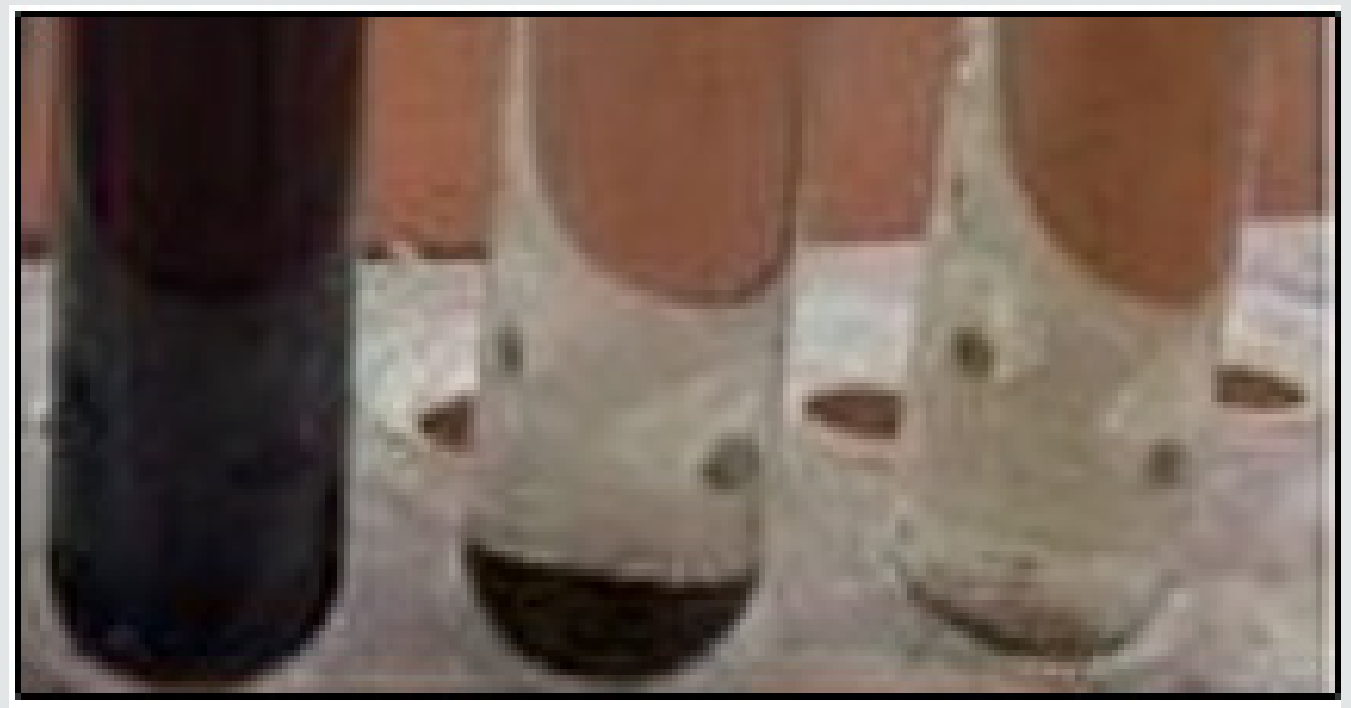

Figure 6: The solubility picture of the nanohydrogel. 


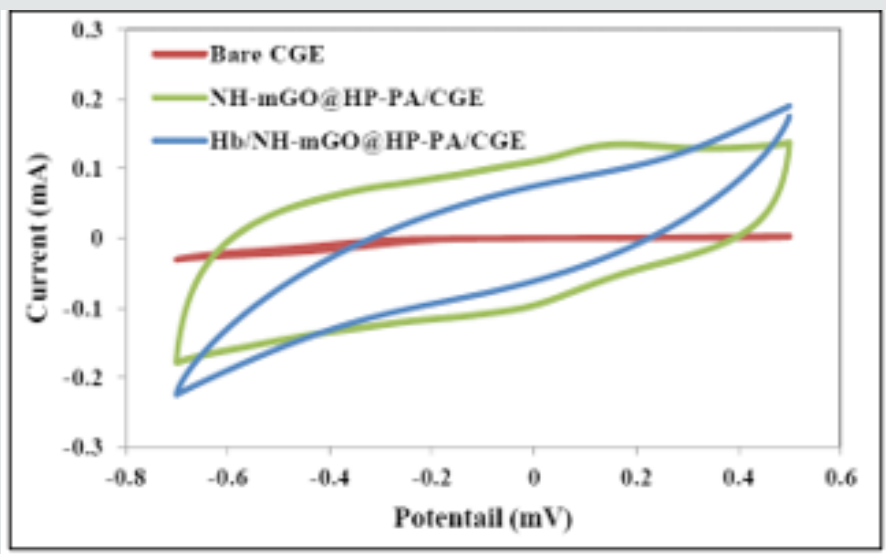

Figure 7: The cyclic voltammograms of the prepared CGEs.

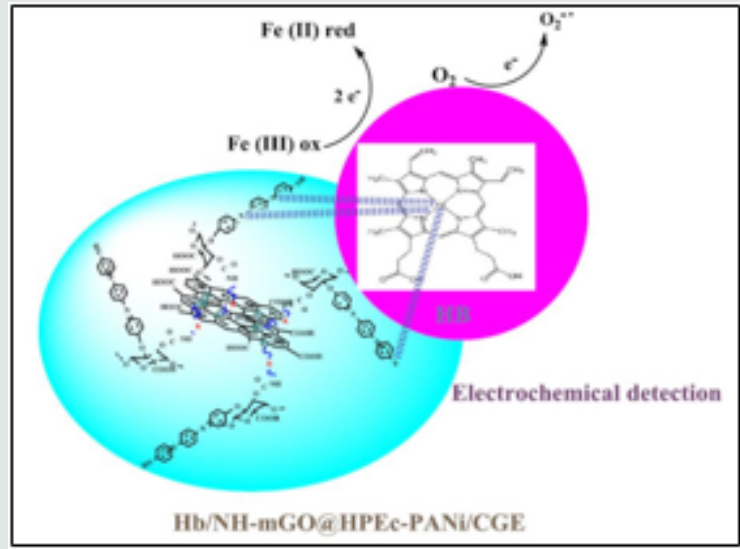

Figure 8: The plausible mechanism of biosensor activity of the Hb/NH-mGOHPEc-PANi/CGE electrode.

\section{Highlight}

A nanohydrogel biomaterial of graphene oxide/pectin/ polyaniline was synthesized. Thermal stability and electrical conductivity were increased in the NH-mGO@HP-PA nanocomposite. Electrodeposition of the nanocomposite enhanced the cyclic voltammetry. Biosensor showed the electrocatalytic activity of nanocomposite to detection of hemoglobin.

\section{Conclusion}

In the present work a nano biomaterial based on HP, PA and GO was synthesized via chemical modification and polymerization. The samples were characterized via FTIR, XRD, SEM, and TGA/ DTA analyses. The nanocomposite showed an enhanced electrical conductivity relative to pure PA. The SEM images showed a nano spherical/laminate morphology for nano biomaterial. The TGA/ DTA analysis indicated higher thermal stability for the NH-mGO@ HP-PA nanocomposite compared to pure GO. The biomaterial was studied in terms of biosensing application for detection of hemoglobin. The NH-mGO@HP-PA/CGE electrode showed an enhanced cyclic voltammetry in the presence of the nanocomposite due to the increase of electron transfer and a reduction in the redox currents due to the cover of enzyme to the PANi coating on the surface of electrode which indicating the sensitivity of the $\mathrm{NH}$ mGO@HP-PA/CGE to hemoglobin detection.

\section{Acknowledgement}

We appreciate the deputy of finance of university for partial supporting this work.

\section{Conflict of Interest}

There are no conflict of interest to declare.

\section{References}

1. Yang A, Li J, Zhang C, Zhang W, Ma N (2015) One-step amine modification of graphene oxide to get a green trifunctional metal-free catalyst. Applied Surface Science 346: 443-450.

2. Stankovich S, Piner RD, Nguyen ST, Ruoff RS (2006) Synthesis and exfoliation of isocyanate-treated graphene oxide nanoplatelets. Carbon 44(15): 3342-3347.

3. Tang L, Shim JJ (2013) Electrochemical property of graphene oxide/ polyaniline composite prepared by in situ interfacial polymerization. Colloid and Polymer Science 291(9): 2237-2243. 
4. Xu J, Wang K, Zu SZ, Han BH, Wei Z(2010) Hierarchical nanocomposites of polyaniline nanowire arrays on graphene oxide sheets with synergistic effect for energy storage. ACS nano 4(9): 5019-5026.

5. Zhang Q, Li Y, Feng Y, Feng W (2013)Electro polymerization of graphene oxide/polyaniline composite for high-performance supercapacitor. Electrochimica Acta 90: 95-100.

6. Ma L, Su L, Zhang, J, Zhao D, Qin C et al. (2016) A controllable morphology GO/PANI/metal hydroxide composite for supercapacitor. Journal of Electroanalytical Chemistry 777: 75-84.

7. Zheng J, Ma X, He X, Gao M, Li, G (2012)Preparation, characterizations, and its potential applications of PANi/graphene oxide nanocomposite. Procedia engineering 27: 1478-1487.

8. Krishnan SK, Singh E, Singh P, Meyyappan M, Nalwa HS (2019) A review on graphene-based nanocomposites for electrochemical and fluorescent biosensors. RSC advances 9(16): 8778-8881.

9. Bora C,Dolui SK (2012) Fabrication of polypyrrole/graphene oxide nanocomposites by liquid/liquid interfacial polymerization and evaluation of their optical, electrical and electrochemical properties. Polymer 53(4): 923-932.
10. Dhand C, Das M, Datta M, Malhotra BD (2011) Recent advances in polyaniline-based biosensors. Biosensors and Bioelectronics 26(6): 2811-2821.

11. Radhapyari K,Kotoky P, Das MR, Khan R (2013) Graphene-polyaniline nanocomposite-based biosensor for detection of antimalarial drug artesunate in pharmaceutical formulation and biological fluids. Talanta 111: 47-53.

12. Bo Y, Yang H, Hu Y, Yao T, Huang S (2011) A novel electrochemical DNA biosensor based on graphene and polyaniline nanowires. Electrochimica Acta 56(6): 2676-2681.

13. Hu XW, Mao CJ, Song JM,Niu HL, Zhang SY et al. (2013) Fabrication of $\mathrm{GO} / \mathrm{PANi} / \mathrm{CdSe}$ nanocomposites for sensitive electrochemiluminescence biosensor. Biosensors and Bioelectronics 41: 372-378.

14. Xu Q, Gu SX,Jin L, Zhou YE, Yang Z et al. (2014) Graphene/polyaniline/ gold nanoparticles nanocomposite for the direct electron transfer of glucose oxidase and glucose biosensing. Sensors and Actuators B: Chemical 190: 562-569.

15. Chen J, Liu W, Liu CM, Li T, Liang RH et al. (2015) Pectin modifications: A review. Critical reviews in food science and nutrition 55(12): 16841698.

\section{(c) (P) \\ This work is licensed under Creative Commons Attribution 4.0 License}

To Submit Your Article Click Here:

Submit Article
DOI: $10.32474 /$ MAMS.2020.02.000148

Citation: Moslem M L, Azita A, Mohsen N, Reza O. Fabrication of a Nanocomposite Based on Pectin/Polyaniline/Graphene Oxide as Novel Electrically Conductive Biomaterial for Biosensing Detection of Hemoglobin. Mod App Matrl Sci 2(5)- 2020. MAMS.MS.ID.000148. DOI: 10.32474/MAMS.2020.02.000148.

\section{Modern Approaches on Material Science}

\section{Assets of Publishing with us}

- Global archiving of articles

- Immediate, unrestricted online access

- Rigorous Peer Review Process

- Authors Retain Copyrights

- Unique DOI for all articles 